IFT-UAM/CSIC-01-25; FTUAM-01/17

\title{
Standard Model Engineering with Intersecting Branes ${ }^{a}$
}

\author{
Luis E. Ibáñez \\ Departamento de Física Teórica $C$-XI and Instituto de Física Teórica C-XVI, \\ Universidad Autónoma de Madrid, Cantoblanco, 28049 Madrid, Spain
}

\begin{abstract}
I briefly describe the recent construction of the first specific string D-brane models yielding just the SM massless fermion spectrum. One of the most remarkable facts predicted by these constructions is that the global symmetries of the SM like Baryon and Lepton numbers as well as the axial $U(1)_{A}$ are gauged symmetries whose anomalies are cancelled by a Green-Schwarz mechanism only in the case of three quark-lepton generations. Proton stability and the Dirac nature of neutrino masses are thus a prediction of these theories. This is work done in collaboration with Fernando Marchesano and Raul Rabadán.
\end{abstract}

Since 1984 a lot of effort has been devoted to the construction of string compactifications yielding a spectrum as close as possible to the Standard Model (SM). A number of explicit three-generation models have been constructed and other important phenomenological questions have been addressed. In spite of these successes I think it is fair to say that up to now there is not a completely satisfactory model. The models in general have a tendency to produce extra unwanted gauge interactions and extra fermions beyond the minimal content of the SM (or the MSSM). Although some models are argued to get closer to the observed physics their analysis requires a very specific and arbitrary choice of scalar flat directions. Most importantly, crucial properties like proton stability depend on these particular choices of scalar vacua.

One of the important difficulties in building realistic models from the perturbative heterotic string was the necessity of following a top-bottom approach to model building: one starts from a theory in higher dimensions and a large gauge group (e.g., $E_{8} \times E_{8}$ ) and breaks the symmetry down to $S U(3) \times S U(2) \times U(1)$ as well as one can. This procedure has as a consequence the proliferation of extra fermions and gauge symmetries beyond the searched SM.

Our new understanding of string theory has changed the situation drastically. We know now that the Dp-branes, solitonic sectors of Type II string theory, are the natural objects when discussing gauge interactions. Dp-branes

${ }^{a}$ To appear in the proceedings of SUSY-01, Dubna (Russia), June 2001. 
have gauge (and matter) particles living on a $(p+1)$ submanifold of the whole 10 (or 11) dimensions. A set of $N$ parallel Dp-branes generically have a $U(N)$ gauge theory living on their worldvolume. Thus we have now the ingredients to follow a bottom-up approach to the construction of a realistic string model: we just have to consider some stacks of D-branes for the different SM gauge groups $S U(3), S U(2)$ and $U(1)$. Unfortunately life is not so easy: the gauge theories on the worldvolume of Dp-branes are non-chiral. For example, $M$ parallel D3-branes yield a 4-dimensional gauge theory with $N=4$ supersymmetry.

In order to get a chiral theory on the worldvolume of D-branes there are not that many options known at the moment. One of the simplest is locating the (9-p) transverse coordinates of the considered brane on top of some singularity in extra dimensions 1 . For example, locating D3-branes on top of an orbifold $C^{3} / Z_{N}$ singularity. This system of branes $f^{2}$ be embedded in a variety of manners in a compact 6 -dimensional space ${ }^{3}$ and one can get semi-realistic models with three generations of quarks and leptons of the SM or left-right symmetric models. This new classes of models are interesting in their own right and provide explicit examples of semi-realistic brane-world theories with gravity mediated SUSY and three generations 3 .

The other known alternative in order to get chiral fermions on the worldvolume of D-branes is intersecting branest. Consider for example a bunch of $\mathrm{N}$ Dp-branes and another set of M Dp-branes $(p>3)$ containing both Minkowski space and intersecting at some angle in the (p-3) extra dimensions. Then one can check that in general one will get massless chiral fermions transforming like $(N, \bar{M})$ under the gauge group $U(N) \times U(M)$. This is quite interesting because the SM is a product of unitary groups and quarks and leptons may be considered to transform under bifundamental representations. In addition there is a very interesting property: if the extra 6 dimensions are compact, the intersection of a couple of branes is in general multiple 0 . 20 . Thus in the context of intersecting branes the replication of generations is natural. This structure to get a SM spectrum is quite general. Some simple examples may be obtained by considering $\mathrm{D}(3+\mathrm{n})$ branes wrapping on the compact space $T^{2 n} \times\left(T^{2(3-n)}(Z)\right.$, for $n=1,2,3$ and intersecting at angles in the $T^{2 n}$ (see

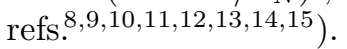

Recently a particularly interesting class of models yielding just the massless fermion spectrum of the SM was constructed 12 . Let me summarize the main features of these constructions. We consider Type IIA string theory compactified on a six-torus $T^{2} \times T^{2} \times T^{2}$. Because of technical reasons to be explained below we do an "orientifold" twist 16 of this theory by the product $\Omega \times R$, where $\Omega$ is the worldsheet parity operator and $R$ is the reflection oper- 
ator with respect of one of the axis of each tori. Now we consider D6-branes containing inside Minkowski space and wrapping each of the three remaining dimensions 8 of the branes on a different torus $T^{2}$. We will denote by $\left(n_{a}^{i}, m_{a}^{i}\right)$, $\mathrm{i}=1,2,3$ the wrapping numbers of each brane $D 6_{a}, n_{a}^{i}\left(m_{a}^{i}\right)$ being the number of times the brane is wrapping around the $\mathrm{x}(\mathrm{y})$-coordinate of the $i-t h$ torus. One can check that the number of times two branes $D 6_{a}$ and $D 6_{b}$ intersect in $T^{6}$ is given by the intersection number $\mathrm{B}$ :

$$
I_{a b}=\left(n_{a}^{1} m_{b}^{1}-m_{a}^{1} n_{b}^{1}\right)\left(n_{a}^{2} m_{b}^{2}-m_{a}^{2} n_{b}^{2}\right)\left(n_{a}^{3} m_{b}^{3}-m_{a}^{3} n_{b}^{3}\right)
$$

Open strings stretching around the intersections give rise to chiral fermions in the bifundamental representation $\left(N_{a}, \bar{N}_{b}\right)$ under the gauge group of the two branes $U\left(N_{a}\right) \times U\left(N_{b}\right)$. Thus these configurations yield $I_{a b}$ copies of the same bifundamental representation, providing a natural source for the observed generation replication. Let us now show how to build a fenfiguration giving just three generations of a $S U(3) \times S U(2) \times U(1)_{Y}$ group 12. We will consider four stacks of branes with multiplicities $N_{a}=3, N_{b}=2, N_{c}=1, N_{d}=1$, giving thus rise to an initial gauge group $U(3) \times U(2) \times U(1) \times U(1)$. Now, it is easy to find wrapping numbers $\left(n_{a}^{i}, m_{a}^{i}\right)$ for the four sets of D6-branes yielding the desired SM spectrum. It is enough to select them in such a way that the intersection numbers $I_{i j}, i, j=a, b, c, d$ are given by 12

$$
\begin{aligned}
& I_{a b}=1 ; I_{a b *}=2 \\
& I_{a c}=-3 ; I_{a c *}=-3 \\
& I_{b d}=0 ; I_{b d *}=-3 \\
& I_{c d}=-3 ; I_{c d *}=3
\end{aligned}
$$

all other intersections vanishing. Here a negative number denotes that the corresponding fermions should have opposite chirality to those with positive intersection number. The massless fermion spectrum living at the intersections is shown in Table 1, as well as the charges with respect to the four $U(1)$ 's. Note that, due to the orientifold operation $\Omega R$ one has to include the D6branes which are "mirror" under that operation and have the same wrapping numbers except for a flip in sign for the $m_{a}^{i}$ 's. We denote the mirror D6-branes with a star. It turns out that the intersection of a $D 6_{a}$ brane with the mirror of a $D 6_{b}$ brane gives rise to a bifundamental of the type $\left(N_{a}, N_{b}\right)\left(\operatorname{not}\left(N_{a}, \bar{N}_{b}\right)\right)$. Note that the left-handed quarks in the table are not universal under the $U(1)_{b}$ charge. This is because one of them comes from the intersection $(a b)$ whereas

${ }^{b}$ Although apparently such a structure would yield four gauged $U(1)$ 's, we show below that we expect three of these $U(1)$ 's to become massive and decouple from the low-energy spectrum. 
the other two come from intersections $\left(a b^{*}\right)$. This is forced upon us if we want to have a consistent theory and this is why we did the orientifold twist to begin with. In all D-brane models strong constraints appear from cancellation of Ramond-Ramond (RR) tadpoles which in turn guarantees the cancellation of gauge anomalies. In the case of the D-brane models here discussed tadpole cancellation requires that there should be as many $N_{a}$ as $\bar{N}_{a}$ representations for any $U\left(N_{a}\right)$ group. In our particular case this lack of universality of left-handed quarks is required in order to have as many $U(2)$ doublets as anti-doublets, as the reader may easily check. Note also that they cancel because the number of generations is equal to the number of colours 12 . This connection between number of colours and numbers of generations in this class of models is quite appealing. Note also that the above constraints also require the presence of right-handed neutrinos.

\begin{tabular}{|c|c|c|c|c|c|c|c|}
\hline Intersection & Matter fields & & $Q_{a}$ & $Q_{b}$ & $Q_{c}$ & $Q_{d}$ & $\mathrm{Y}$ \\
\hline \hline$(\mathrm{ab})$ & $Q_{L}$ & $(3,2)$ & 1 & -1 & 0 & 0 & $1 / 6$ \\
\hline$\left(\mathrm{ab}^{*}\right)$ & $q_{L}$ & $2(3,2)$ & 1 & 1 & 0 & 0 & $1 / 6$ \\
\hline$(\mathrm{ac})$ & $U_{R}$ & $3(\overline{3}, 1)$ & -1 & 0 & 1 & 0 & $-2 / 3$ \\
\hline$(\mathrm{ac} *)$ & $D_{R}$ & $3(\overline{3}, 1)$ & -1 & 0 & -1 & 0 & $1 / 3$ \\
\hline$\left(\mathrm{bd}^{*}\right)$ & $L$ & $3(1,2)$ & 0 & -1 & 0 & -1 & $-1 / 2$ \\
\hline$(\mathrm{cd})$ & $E_{R}$ & $3(1,1)$ & 0 & 0 & -1 & 1 & 1 \\
\hline$\left(\mathrm{cd}^{*}\right)$ & $N_{R}$ & $3(1,1)$ & 0 & 0 & 1 & 1 & 0 \\
\hline
\end{tabular}

Table 1: Standard model spectrum and $U(1)$ charges

In ref 12 we gave a general solution for the wrapping numbers $\left(n_{a}^{i}, m_{a}^{i}\right)$ giving rise to a SM spectrum. In here we will limit ourselves to a specific example for illustrative purposes. Consider the wrapping numbers given in Table 2 for our four SM branes 9 The reader can easily check using eq.(11) that these wrapping numbers give rise to the intersection numbers in eq.(2) and hence to the SM spectrum of Table 1.

The analysis of the $U(1)$ gauge symmetries in these models is quite interesting. The four $U(1)$ symmetries $Q_{a}, Q_{b}, Q_{c}$ and $Q_{d}$ have clear interpretations in terms of known global symmetries of the standard model. Indeed $Q_{a}$ is $3 B, B$ being the baryon number and $Q_{d}$ is nothing but (minus)lepton number. Concerning $Q_{c}$, it is twice $I_{R}$, the third component of right-handed weak isospin familiar from left-right symmetric models. Finally $Q_{b}$ has the

${ }^{c}$ The effective fractional $m_{a}^{3}$ winding numbers in the third torus come from the addition of a NS P-field background which is required to get odd number of generations in the orientifold case 11 


\begin{tabular}{|c||c|c|c|}
\hline$N_{i}$ & $\left(n_{i}^{1}, m_{i}^{1}\right)$ & $\left(n_{i}^{2}, m_{i}^{2}\right)$ & $\left(n_{i}^{3}, m_{i}^{3}\right)$ \\
\hline \hline$N_{a}=3$ & $(1,0)$ & $(2,1)$ & $(1,1 / 2)$ \\
\hline$N_{b}=2$ & $(0,-1)$ & $(1,0)$ & $(1,3 / 2)$ \\
\hline$N_{c}=1$ & $(1,3)$ & $(1,0)$ & $(0,1)$ \\
\hline$N_{d}=1$ & $(1,0)$ & $(0,-1)$ & $(1,3 / 2)$ \\
\hline
\end{tabular}

Table 2: Example of D6-brane wrapping numbers giving rise to a SM spectrum.

properties of a Peccei-Quinn symmetry (it has mixed $S U(3)$ anomalies). We thus learn that all these known global symmetries of the SM are in fact gauge symmetries in this class of theories. This is important because it implies that Baryon and Lepton symmetries are gauged symmetries in this class of theories. As a consequence protons are perturbatively stable and neutrino masses must be $\operatorname{Dirac}^{d}$.

Two out of the four $U(1)$ 's have triangle anomalies which are cancelled by a generalized Green-Schwarz mechanism 17,18. The anomalous symmetries are the $Q_{b}$ generator and the $3 Q_{a}-Q_{d}$ generator which can be identified with the $(3 B+L)$. In these toroidal models there are four two-index antisymmetric tensors $B_{2}^{k}, k=0,1,2,3$ which come from the Ramond-Ramond closed string sector of the theory. They have $B_{2} \wedge F^{j}$ type couplings with the four $U(1)$ 's of the theory. Those can be computed and in the example above they are as follows 12.

$$
\begin{aligned}
& B_{2}^{1} \wedge(-2) F^{b} \\
& B_{2}^{2} \wedge 3\left(3 F^{a}-F^{d}\right) \\
& B_{2}^{3} \wedge\left(3 F^{a}+F^{c}\right)
\end{aligned}
$$

whereas the $B_{2}^{0} \mathrm{RR}$ field has no couplings to the $F^{j}$. The antisymmetric $B_{2}^{k}$ fields are dual in four dimensions to pseudoscalars $C_{0}^{k}$. The duals to $B_{2}^{1}$ and $B_{2}^{2}$ have couplings to the full gauge group of the form:

$$
\frac{1}{2} C_{0}^{1}\left(F^{a} \wedge F^{a}-3 F^{d} \wedge F^{d}\right)
$$

${ }^{d}$ Notice this implies that cosmological baryogenesis can only happen at the non-perturbative level, as in weak-scale baryogenesis scenarios. 


$$
\frac{1}{2} C_{0}^{2}\left(-F^{b} \wedge F^{b}+2 F^{c} \wedge F^{c}\right)
$$

whereas the RR scalar $C_{0}^{3}$ does not couple to any $F \wedge F$ term. It is easy to check that the combination of these couplings cancel all $U(1)$ anomalies by the tree-level exchange of the $B_{2}^{k}\left(C_{0}^{k}\right) k=1,2$ fields in the usual way. At the same time, the first two $B \wedge F$ couplings in eq.(3) give a mass of order the string scale $M_{s}$ to the gauge bosons corresponding to the anomalous combinations : $Q_{b}$ and $\left(3 Q_{a}-Q_{d}\right)$. Indeed, after a duality transformation the $B \wedge F$ couplings turn into Higgs-like couplings of type $M_{s}^{2}\left(\partial_{\mu} C_{o}^{k}\right) A_{a}^{\mu}$ which render those $U(1)$ 's massive. In addition something interesting also happens ${ }^{2} 2$ : the third linear combination $\left(3 Q_{a}+Q_{c}\right)$ which is anomaly-free also becomes massive by swallowing the $B_{2}^{3}$ field in a similar way 1 . Thus at the end of the day only the orthogonal linear combination $Q_{Y}=(1 / 6) Q_{a}-(1 / 2) Q_{c}+(1 / 2) Q_{d}$, which is nothing but hypercharge, remains massless $t$. Thus, as promised, the actual gauge group of the theory is just $S U(3) \times S U(2) \times U(1)_{Y}$.

These D6-brane models are non-supersymmetric due to the presence of the non-trivial intersections. Thus, for example, associated to each of the intersections there are massive scalar fields which in some sense may be considered "SUSY-partners", squarks and sleptons, of the massless chiral fermions, have the same multiplicity $\left|I_{i j}\right|$ and carry the same gauge quantum numbers. The lightest of those states have masses 9

$$
\begin{aligned}
& t_{1}: \alpha^{\prime}(\text { Mass })^{2}=\frac{1}{2}\left(-\vartheta_{1}+\vartheta_{2}+\vartheta_{3}\right) \\
& t_{2}: \alpha^{\prime}(\text { Mass })^{2}=\frac{1}{2}\left(\vartheta_{1}-\vartheta_{2}+\vartheta_{3}\right) \\
& t_{3}: \quad \alpha^{\prime}(\text { Mass })^{2}=\frac{1}{2}\left(\vartheta_{1}+\vartheta_{2}-\vartheta_{3}\right)
\end{aligned}
$$

Here $\vartheta_{i}$ are the intersection angles (in units of $\pi$ ) at each of the three subtorus. As is obvious from these formulae the masses depend on the angles at each intersection and hence on the relative size of the radii. Although in principle some of the scalars could be tachyonic, in general it is possible to vary the compact radii in order to get rid of all tachyons of a given model (see 12 ). Although the spectrum at the intersections has no supersymmetry, in the bulk of the D6-branes there is at the tree level $N=4$ SUSY gauge multiplets of each of the groups of the SM. However, loop effects involving the fields at the intersections render the charged SUSY partners of the gauge bosons massive

${ }^{e}$ We are used to the fact that anomalous $U(1)$ 's become massive in $\mathrm{D}=4$ string models. This is something new: now we see that anomaly-free $U(1)$ 's may also become massive due to the generic presence of $B \wedge F$ couplings. Note also that in the case of a low string scale $M_{s}$ the physical SM $Z^{0}$ may owe part of its mass to a $B \wedge F^{Z^{0}}$ term dye to a mixing of order $M_{W} / M_{s}$ with the other $U(1)$ 's, both anomalous and non-anomalous 19 .

${ }^{f}$ The massive $U(1)$ 's remain however as effective global symmetries. 
and of order the string scale 2 . Other Kaluza-Klein and winding copies of the gauge multiplets appear at the massive level.

Up to now we have ignored the existence or not of the Higgs system required for the breaking of the electroweak symmetry as well as to give masses to quarks and leptons. Looking at the $U(1)$ charges of quarks and leptons in Table 1, we see that possible Higgs fields coupling to quarks come in four varieties with charges under $Q_{b}, Q_{c}$ and hypercharge given in Table 3. Now,

\begin{tabular}{|c|c|c|c|}
\hline Higgs & $Q_{b}$ & $Q_{c}$ & $\mathrm{Y}$ \\
\hline \hline$h_{1}$ & 1 & -1 & $1 / 2$ \\
\hline$h_{2}$ & -1 & 1 & $-1 / 2$ \\
\hline \hline$H_{1}$ & -1 & -1 & $1 / 2$ \\
\hline$H_{2}$ & 1 & 1 & $-1 / 2$ \\
\hline
\end{tabular}

Table 3: Electroweak Higgs fields

the question is whether for some configuration of the branes such Higgs fields appear in the light spectrum. Indeed that is the case. The $U(2)$ branes $\left(b, b^{*}\right)$ are parallel to the $\left(c, c^{*}\right)$ branes along the second torus and hence they do not intersect. However there are open strings which stretch in between both sets of branes and which lead to light scalars when the distance $Z_{2}$ in the second torus is small. The $H_{i}$ 's come from the $b-c^{*}$ intersections whereas the $h_{i}$ come from the $b-c$ intersections. The quadratic scalar potential for these fields has the form:

$$
\begin{array}{r}
V_{2}=m_{H}^{2}\left(\left|H_{1}\right|^{2}+\left|H_{2}\right|^{2}\right)+m_{h}^{2}\left(\left|h_{1}\right|^{2}+\left|h_{2}\right|^{2}\right)+ \\
+m_{B}^{2} H_{1} H_{2}+\text { h.c. }+m_{b}^{2} h_{1} h_{2}+\text { h.c. }
\end{array}
$$

The mass parameters of the potential have an interesting geometrical interpretation in terms of the brane distances and intersection angles and massless scalars appear for appropriate values of the parameters. The number of light Higgs multiplets will be given by the intersection number of the (bc) and ( $\left.\mathrm{bc}^{*}\right)$ branes in the first and third tori, i.e., the number of $h_{i}$ fields is given by $I_{b c}=\left(n_{b}^{1} m_{c}^{1}-m_{b}^{1} n_{c}^{1}\right)\left(n_{b}^{3} m_{c}^{3}-m_{b}^{3} n_{c}^{3}\right)$ and the number of $H_{i}$ fields is given by $I_{b c *}=\left(n_{b}^{1} m_{c}^{1}+m_{b}^{1} n_{c}^{1}\right)\left(n_{b}^{3} m_{c}^{3}+m_{b}^{3} n_{c}^{3}\right)$. In the example above one has $\left|I_{b c}\right|=\left|I_{b c *}\right|=1$ and there is just one Higgs sector of each type. Models in which only one copy of the $H_{i}$ or $h_{i}$ fields appear may be obtained.

There is no room here to describe gther phenomenological aspects of these theories and we refer the reader to ref 12 . Let us just make a few general remarks. In this class of theories each quark and lepton resides on a different 
point in the six extra dimensions. Since in order to get Yukawa couplings the worldsheet has to stretch between different locations for the different generations, one expects the natural appearance of hierarchical Yukawa couplings 1012. Neutrino masses are special in this respect. In the present models $L$ is an exact symmetry in perturbation theory and hence only Dirac masses are allowed. One possibility to obtain sufficiently small neutrino masses is to make use of the above mentioned mechanism to get hierarchies of Yukawa couplings. Neutrino Yukawa couplings could be exponentially suppressed in these theories. Perhaps a more interesting possibility is the case in which there are no Yukawa couplings at all for neutrinos. That is precisely the case if only Higgs fields of type $H_{i}$ above are present. In that case there are no perturbative masses for the lightest quarks nor the neutrinos. However there are in general dimension 6 operators of the form $\alpha^{\prime}\left(L N_{R}\right)\left(Q_{L} U_{R}\right)^{*}$. These come from the exchange of massive string states and are consistent with all gauge symmetries. Plugging the u-quark chiral condensate, neutrino masses of order $12 m_{\nu} \propto\left(<u_{R} u_{L}>\right) / M_{s}^{2}$ are obtained. For $<u_{R} u_{L}>\propto(200 \mathrm{MeV})^{3}$ and $M_{s} \propto 1-10 \mathrm{TeV}$ one gets neutrino masses of order $0.01-1 \mathrm{eV}$ 's, consistent with oscillation experiments. Notice that the dimension 6 operators may have different coefficients for different neutrino generations so there will be in general non-trivial generation structure. We believe that the presence of Dirac neutrino masses of this order of magnitude from this mechanism looks like a general property of low string scale models.

These models are non-supersymmetric and to avoid the hierarchy problem one should shose the string scale to be not much above the weak scale. As noted in ref 8 , the usual procedure 20 for lowering the string scale down to 1-10 $\mathrm{TeV}$ while maintaining the four-dimensional Plank mass at its experimental value cannot be applied directly to these D6-brane toroidal models. This is because if some of the compact radii $R_{1,2}^{i}, i=1,2,3$ are made large some charged KK modes living on the branes would become very light. In refl it was proposed a way in which one can have a low string scale compatible with the four-dimensional large Planck mass. The idea is that the 6 -torus could be small while being connected to some very large volume manifold. For example, one can consider a region of the 6-torus away from the D6-branes, cut a ball and gluing a throat connecting it to a large volume manifold. In this way one would obtain a low string scale model without affecting directly the brane structure discussed above. Alternatively one can construct analogous models 21 in terms of D5-branes wrapping on $T^{4} \times T^{2} / Z_{N}$. In this case the standard mechanism to lower the string scale can be obtained by making large the two dimensions transverse to the D5-branes $\mathrm{O}_{2} 21$.

In summary, present day knowledge of string theory in terms of branes 
allow us to follow a bottom-up approach to the string embedding of the SM. Simple configurations of intersecting branes allow us for the first time the construction of brane configurations with massless fermion sector identical to that of the non-SUSY SM (see ref14 for a recent attempt for the SUSY case). Interestingly enough, such constructions imply the gauging of the perturbative symmetries of the SM like baryon and lepton number. In our opinion this provides a neat and beautiful explanation of the surprising stability of the proton. Furthermore, anomaly cancellation seems to work in this context only due to the fact that the number of colours in the SM is equal to the number of generations, offering a nice explanation for the generation puzzle.

The different sizes of the SM gauge couplings in these theories have to do with the different volumes on which each different D-brane is wrapping, and one can vary those volumes so that the observed gauge couplings are reproduced. Thus the logarithmic unification of couplings will be lost, like in any model with a low string scale. In any event, we physicists cannot rely on a single piece of data as MSSM gauge coupling unification is. It could well be that the nice agreement of gauge coupling unification in the MSSM could be fortuitous. Recall: the apparent size of the sun agrees with that of the moon with a good precission. For centuries mankind has given special meaning to this "size unification" which turns out to be just an accident in the formation of the solar system. Perhaps we should learn the lesson.

\section{Acknowledgements}

I am grateful to my collaborators G. Aldazabal, S. Franco, F. Marchesano, R. Rabadán and A . Uranga for a very enjoyable collaboration on topics discussed in this talk. This work is partially supported by CICYT (Spain) and the European Commission (RTN contract HPRN-CT-2000-00148).

1. M. R. Douglas, B. R. Greene, D. R. Morrison, Nucl. Phys. B506 (1997) 84, hep-th/9704151; S. Kachru, E. Silverstein, Phys. Rev. Lett. 80 (1998) 4855, hep-th/9802183; A. Lawrence, N. Nekrasov, C. Vafa, Nucl. Phys. B533 (1998) 199.

2. G. Aldazabal, L. E. Ibáñez, F. Quevedo, A. M. Uranga, JHEP 0008:002,2000. hep-th/0005067

3. G. Aldazabal, L. E. Ibáñez, F. Quevedo, JHEP 0001 (2000) 031, hepth/9909172, JHEP02 (2000) 015, hep-ph/0001083.

4. A. Uranga, hep-th/0007206.

5. D. Bailin, G. Kraniotis and A Love, hep-th/0011289.

6. D. Berenstein, V. Jejjala and R.G. Leigh, hep-ph/0105042. 
7. M. Berkooz, M. R. Douglas, R. G. Leigh, Nucl.Phys. B480(1996)265, hep-th/9606139.

8. Ralph Blumenhagen, Lars Goerlich, Boris Kors, Dieter Lust, JHEP 0010 (2000) 006, hep-th/0007024; hep-th/0010198.

9. G. Aldazabal, S. Franco, L.E. Ibañez, R. Rabadán, A.M. Uranga, hepth/0011073.

10. G. Aldazabal, S. Franco, L.E. Ibañez, R. Rabadán, A.M. Uranga, hep$\mathrm{ph} / 0011132$.

11. Ralph Blumenhagen, Boris Kors, Dieter Lust, JHEP 0102 (2001) 030, hep-th/0012156.

12. L. Ibáñez, F. Marchesano and R. Rabadán, hep-th/0105155.

13. R. Blumenhagen, B. Kors, D. Lust and T. Ott, hep-th/0107138.

14. M. Cvetic, G. Shiu and A. Uranga, hep-th/0107143; hep-th/0107166.

15. D. Bailin, G. Kraniotis and A. Love, hep-th/0108131.

16. A. Sagnotti, in Cargese 87, Strings on Orbifolds, ed. G. Mack et al. (Pergamon Press, 1988) p. 521; P. Horava, Nucl. Phys. B327 (1989) 461; J. Dai, R. Leigh and J. Polchinski, Mod.Phys.Lett. A4 (1989) 2073; G. Pradisi and A. Sagnotti, Phys. Lett. B216 (1989) 59 ; M. Bianchi and A. Sagnotti, Phys. Lett. B247 (1990) 517 ; Nucl. Phys. B361 (1991) 519 .

17. M. Dine, N. Seiberg and E. Witten, Nucl. Phys. B289 (1987) 589; J. Atick, L. Dixon and A. Sen, Nucl. Phys. B292 (1987) 109; M. Dine, I. Ichinose and N. Seiberg, Nucl. Phys. B293 (1987) 253.

18. L. E. Ibáñez, R. Rabadán, A. M. Uranga, Nucl.Phys. B542 (1999) 112138 , hep-th/9808139.

19. L.E. Ibáñez and F. Quevedo, in progress.

20. N. Arkani-Hamed, S. Dimopoulos and G. Dvali, Phys. Lett. B429 (1998) 263, hep-ph/9803315;

I. Antoniadis, N. Arkani-Hamed, S. Dimopoulos, G. Dvali Phys. Lett. B436 (1999) 257, hep-ph/9804398.

21. D. Cremades, L.E. Ibáñez and F. Marchesano, to appear. 